\title{
Detecting Circumbinary Exoplanets: Understanding Transit Timing
}

\author{
David Armstrong ${ }^{1}$, David V. Martin ${ }^{2}$ and Don Pollacco ${ }^{1}$ \\ ${ }^{1}$ University of Warwick, Department of Physics, Gibbet Hill Road, \\ Coventry, CV4 7AL, UK \\ email: d.j.armstrong@warwick.ac.uk \\ ${ }^{2}$ Observatoire de Genève, Université de Genève, 51 chemin des Maillettes, \\ Sauverny 1290, Switzerland \\ email: david.martin@unige.ch
}

\begin{abstract}
We have derived and tested a simple analytical model for placing limits on the transit timing variations of circumbinary exoplanets. These are generally of days in magnitude, dwarfing those found in multi-planet systems. The derived method is fast, efficient and is accurate to approximately $1 \%$ in predicting limits on the possible times of transits over a 3-year campaign.
\end{abstract}

Keywords. planetary systems: dynamical evolution; binaries: eclipsing; celestial mechanics

\section{Introduction}

The NASA Kepler mission has led to the detection of seven transiting circumbinary exoplanets. Detecting these planets via the transit method presents observational challenges, as they exhibit transit timing variations (TTVs) on the order of days in magnitude, in addition to changes in the transit shape and duration.

The purpose of this work is to present constraints on the observational characteristics of a transiting circumbinary explained, through our knowledge of the host binary, using a fast method which requires no complex modelling. All of the details of this work are in Armstrong \& Martin et al. (2013).

\section{Model}

We present a model which accurately places limits on the magnitude of circumbinary TTVs. An example circumbinary system is depicted in Fig. 1, with solid lines showing the planetary orbital phases which could contain transits, as calculated using a numerical Nbody integrator. The analytical bounds are denoted by two dashed lines. The analytical prediction is composed of two different effects:

Geometrical. This is caused by the binary stars moving through an extended region of space. The planets can hence take several days to traverse the full extent of the binary orbit, and it is during this time that transits will occur. The geometrical effect corresponds to the thickness of the bands in Fig. 1. The thickness will change over time as the planet precesses, since the planet will pass the binary orbit at a speed dependent on its argument of periapse, $\omega_{\mathrm{p}}$. The magnitude of geometrical effect was derived by taking the transit duration equation for a single star and extending it to allow for a moving body.

Precessional. Due to the non point mass nature of the binary, large torques are exerted on the planet's orbit. The main result of this is a swift precession of $\omega_{\mathrm{p}}$. This precession causes the time of potential transits to shift away from the 'expected' time for a constant 


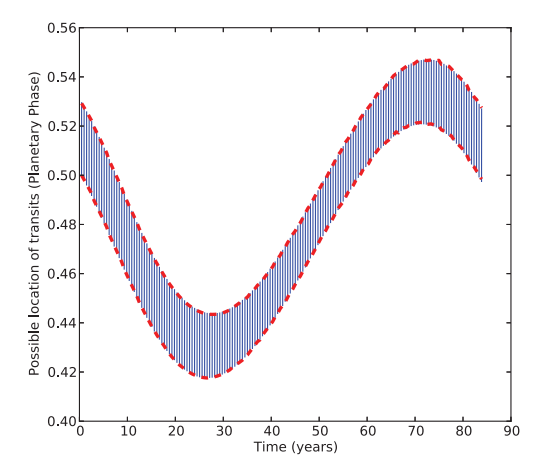

Figure 1. Numerical transit regions (solid lines) bounded by the analytical predictions (dashed lines).

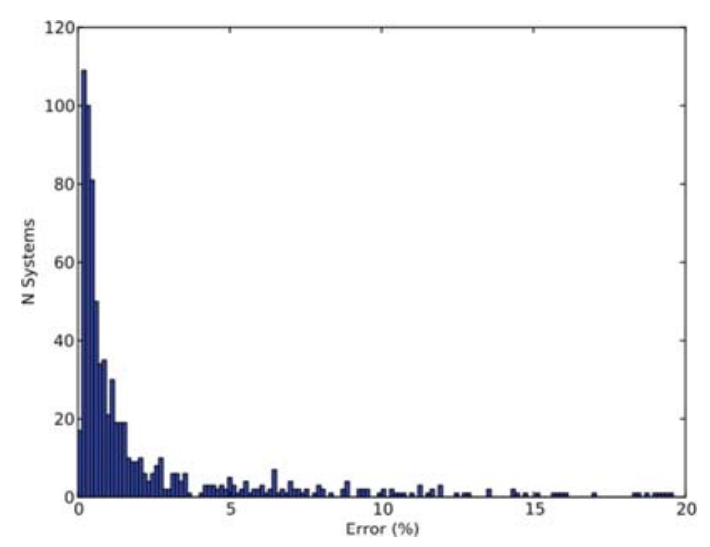

Figure 2. Histogram of the analytical prediction error, as a percentage of the numerical transit region time.

periodic signal. The magnitude of these shifts at a given time depends upon the instantaneous value of $\omega_{\mathrm{p}}$. The precessional effect manifests itself as an oscillatory offset in the transit phase range given by the geometrical variation, as seen in Fig. 1. The precessional effect was modelled using a linear precession rate from Doolin \& Blundell (2011), which was originally derived from an equation in Farago \& Laskar (2010).

\section{Testing}

The accuracy of the model was tested on a simulated distribution of 1,000 single-planet circumbinary systems, 799 of which were stable over 1,200 years. The binary distribution was drawn from a combination of the Kepler survey and the Halbwachs et al. (2003) radial velocity survey. Given the lack of a circumbinary planet distribution, the simulated planets were created based on those found by radial velocity surveys, with an additional stability constraint based on the work of Holman \& Wiegert (1999). A histogram of the simulation errors is in Fig. 2. For clarity, 43 badly-predicted systems with errors higher than $20 \%$ are not shown.

\section{Applications}

The equations derived in Armstrong \& Martin et al. (2013) can be used in searches for circumbinary exoplanets. As a specific example, they can be used to set the parameters $\triangle_{\min }$ and $\triangle_{\max }$ in the QATS search algorithm (Carter \& Agol, 2013). The equations are also useful in reverse, for making first estimates of planet parameters using the observed TTV of a newly discovered candidate.

\section{References}

Armstrong, D. \& Martin, D. V. et al. 2013, MNRAS, 434, 3047

Carter, J. A. \& Agol, E. 2013, ApJ, 765, 132

Doolin, S. \& Blundell, K. M. 2011, MNRAS, 418, 2656

Farago, F. \& Laskar, J. 1993, MNRAS, 401, 1189

Halbwachs, J. L., Mayor, M., Udry, S., Arenou, F. 2003, A $\& A, 397,159$

Holman M. \& Wiegert P. 1999, ApJ, 117, 621 